\title{
Risk factors influencing survival of acellular porcine corneal stroma in infectious keratitis: a prospective clinical study
}

Saiqun $\mathrm{Li}^{\dagger}$, Meng Li ${ }^{\dagger}$, Li Gu, Lulu Peng, Yuqing Deng, Jing Zhong, Bowen Wang, Qian Wang, Yichen Xiao and Jin Yuan ${ }^{*}(\mathbb{0}$

\begin{abstract}
Background: A worldwide lack of donor corneas demands the bioengineered corneas be developed as an alternative. The primary objective of the current study was to evaluate the efficacy of acellular porcine corneal stroma (APCS) transplantation in various types of infectious keratitis and identify risk factors that may increase APCS graft failure.

Methods: In this prospective interventional study, 39 patients with progressive infectious keratitis underwent therapeutic lamellar keratoplasty using APCS and were followed up for 12 months. Data collected for analysis included preoperative characteristics, visual acuity, graft survival and complications. Graft survival was evaluated by the KaplanMeier method and compared with the log-rank test.

Results: The percentage of eyes that had a visual acuity of $20 / 40$ or better increased from $10.3 \%$ preoperatively to $51.2 \%$ at 12 months postoperatively. Twelve patients (30.8\%) experienced graft failure within the follow-up period. The primary reasons given for graft failure was noninfectious graft melting $(n=5)$, and the other causes included recurrence of primary infection $(n=4)$ and extensive graft neovascularization $(n=3)$. No graft rejection was observed during the follow-up period. A higher relative risk (RR) of graft failure was associated with herpetic keratitis ( $R R=8.0$, $P=0.046)$ and graft size larger than $8 \mathrm{~mm}(R R=6.5, P<0.001)$.

Conclusions: APCS transplantation is an alternative treatment option for eyes with medically unresponsive infectious keratitis. Despite the efficacy of therapeutic lamellar keratoplasty with APCS, to achieve a good prognosis, restriction of surgical indications, careful selection of patients and postoperative management must be emphasized.

Trial registration Prospective Study of Deep Anterior Lamellar Keratoplasty Using Acellular Porcine Cornea, NCT03105466. Registered 31 August 2016, ClinicalTrails.gov
\end{abstract}

Keywords: Therapeutic lamellar keratoplasty, Infectious keratitis, Acellular porcine corneal stroma

\section{Background}

Infective keratitis is considered a major global cause of visual impairment [1-3]. In severe and progressive infections that are nonresponsive to medical treatment,

*Correspondence: yuanjincornea@126.com

†Saiqun Li and Meng Li contributed equally to this work State Key Laboratory of Ophthalmology, Zhongshan Ophthalmic Center, Sun Yat-sen University, Guangzhou 510060, China therapeutic keratoplasty serves as a last resort to remove the infective focus or restore globe integrity. Therapeutic keratoplasty, including penetrating keratoplasty (PKP) and anterior lamellar keratoplasty (ALK), contributes to a significant proportion of corneal transplantations performed in the developing world, especially in Asia $[4,5]$. Although keratoplasty works well in infective keratitis, the shortage of donor corneas is the major limiting factor for the performance of corneal transplantation in many

(c) The Author(s) 2019. This article is licensed under a Creative Commons Attribution 4.0 International License, which permits use, sharing, adaptation, distribution and reproduction in any medium or format, as long as you give appropriate credit to the original author(s) and the source, provide a link to the Creative Commons licence, and indicate if changes were made. The images or other third party material in this article are included in the article's Creative Commons licence, unless indicated otherwise in a credit line to the material. If material is not included in the article's Creative Commons licence and your intended use is not permitted by statutory regulation or exceeds the permitted use, you will need to obtain permission directly from the copyright holder. To view a copy of this licence, visit http://creativeco mmons.org/licenses/by/4.0/. The Creative Commons Public Domain Dedication waiver (http://creativecommons.org/publicdomain/ zero/1.0/) applies to the data made available in this article, unless otherwise stated in a credit line to the data. 
countries, which leads to the development of biomimetic and implantable human corneal substitutes [6]. Many methods of stromal replacements have been proposed to date [7-9]. Recently, biosynthetic collagen hydrogel and acellular porcine corneal stroma (APCS) have been tested in a very limited number of human clinical trials with promising results $[10,11]$.

APCS is processed from fresh porcine corneas by removing nuclear and cellular materials [12]. The approved indication for APCS is the treatment of infectious keratitis. However, the effectiveness of APCS has so far been reported only in fungal keratitis and herpes simplex keratitis (HSK) [10, 11]. Therefore, to provide better guidance for clinical practice, we conducted this study to evaluate the efficacy of APCS keratoplasty in progressive infectious keratitis caused by a wide variety of pathogens, such as fungi, bacteria, viruses or Acanthamoeba. In addition, another purpose of the present study was to identify some of the factors that may increase the therapeutic failure rate of APCS transplantation so that future patients with a high risk of graft failure may be excluded and avoidance measures may be taken.

\section{Methods}

\section{Patient population}

Thirty-nine patients (39 eyes) undergoing APCS transplantation in Zhongshan Ophthalmic Center, Sun YatSen University to treat medically uncontrolled infectious keratitis with a bacterial, fungal, Acanthamoeba or viral etiology were consecutively enrolled in this prospective cohort study. Infectious keratitis was defined as medically uncontrolled if the disease had significantly progressed after a 2-week of maximum antimicrobial medication or if the cornea was at risk of perforation. All participants completed 12 months of postoperative follow-up. The diagnosis of nonviral infectious keratitis was achieved by smear, microbial culture, confocal microscopy and pathologic examination. The diagnosis of HSK was based on multiple episodes of recurrence and clinical manifestations (typical epithelial dendritic lesions and stromal abnormalities). The exclusion criteria for APCS transplantation included an infiltration size larger than $9 \mathrm{~mm}$ and signs associated with infection that affected the corneal endothelium and anterior chamber. Hypopyon was not an absolute contraindication for ALK as long as it was due to severe intraocular sterile inflammation. Noninfectious hypopyon was distinguished from infectious hypopyon based on clinical appearance. Compared to infectious hypopyon, a noninfectious hypopyon is generally relatively more likely to shift with a change in head position. However, there is substantial overlap between these two entities. Moreover, if the lesions were noted to not extended to the level of Descemet's membrane (DM), the hypopyon was presumed to be noninfectious. Nonetheless, the early intraocular spread of infection could not be fully excluded. It is even especially difficult to distinguish noninfectious hypopyon from infectious hypopyon in cases with a deep lesion. In light of these considerations, we prudently performed lamellar keratoplasty in cases with hypopyon.

\section{Corneal stroma substitutes}

The APCS graft used in the current study was prepared by AiNear Corneal Engineering Corporation (Shenzhen, China) as previously reported [13]. After it was enucleated from a quarantined pigs immediately after its death, each fresh eyeball was thoroughly washed in sterile phosphate-buffered saline (PBS). Whole corneas with $2 \mathrm{~mm}$ of sclera were cut from the eyeballs using a 16- $\mathrm{mm}$ trephine and immersed into ultrapure water for $12 \mathrm{~h}$ to allow swelling. The corneoscleral tissue was then agitated for three times of $30 \mathrm{~min}$ in $2 \mathrm{M} \mathrm{NaCl}$ followed by $30 \mathrm{~min}$ in ultrapure water. Next, the corneas were washed in $0.2 \%$ Triton X-100 for $6 \mathrm{~h}$ and then thoroughly washed in PBS to remove any remnant of Triton X-100 from the tissue. After it was dehydrated in glycerol until it reached a normal natural corneal thickness, the APCS was trimmed to a diameter of $10 \mathrm{~mm}$ and a thickness of $450 \mu \mathrm{m}$. Finally, sterilization was performed using $\mathrm{Co}^{60}$ radiation.

\section{Histological examination of materials}

APCS and human donor corneas were processed for routine paraffin embedding. Briefly, 5 - $\mu \mathrm{m}$-thick sections were cut and stained with hematoxylin and eosin (H\&E). Tissues were also cut into pieces and processed for scanning electron microscopy (SEM, Hitachi SU8100) examination as described previously [14]. SEM images were obtained at a total magnification of $1000 \times$.

\section{Surgical technique}

Deep anterior lamellar keratoplasty (DALK) surgery was performed in a minimally modified manner as previously described [4]. Briefly, after trephination of approximately $2 / 3$ of the total host corneal thickness using a Hessburg-Barron vacuum trephine, the remaining stroma was removed to a level close to the DM using a manual technique. Baring of DM was achieved in two cases of descemetocele. The recipient bed was $1 \mathrm{~mm}$ larger than the infiltration size. Additionally, in cases with bacterial and fungal keratitis, the exposed recipient bed was intraoperatively irrigated with antibacterial (ceftazidime) or antifungal (fluconazole) agents. Anterior chamber irrigation was performed in cases with sterile hypopyon. Since the APCS was dehydrated for preservation, it needed to be soaked in saline for 1 min before it was used for trephination in keratoplasty. APCS graft buttons were 
oversized by $0.25 \mathrm{~mm}$ and fixed to the recipient bed with interrupted 10-0 nylon sutures (Alcon, Fort Worth, TX).

\section{Postoperative treatment}

To prevent recurrent infection, we postoperatively administrated multiple topical and systemic antimicrobial medications. For nonviral keratitis, systemic antimicrobial treatments [cephalosporin (ceftazidime) or fluoroquinolones (levofloxacin) for bacterial keratitis, according to the causative microbe and drug-susceptibility and voriconazole for fungal keratitis] were administered within 1 to 2 weeks postoperatively, while topical antimicrobial medications were usually maintained for at least 1 month after surgery and then tapered over the ensuing weeks if no infection recurred. For HSK, the postoperative antiviral treatment regimen comprised oral acyclovir $400 \mathrm{mg}$ twice a day for 12 months and topical antiviral medications (ganciclovir and interferon) for at least 3 months. In cases of fungal and Acanthamoeba keratitis, topical steroids were withheld for at least 2 weeks, whereas for bacterial and viral infections, topical steroid treatment was initiated immediately after surgery. Topical steroids were generally prescribed four times daily for 6 months, gradually tapered down to a maintenance dose of once daily over a 3-month period, and then continued for another 3 months. Topical tacrolimus was used right after the surgery as an additional immunosuppressive medication and for its anti-inflammatory effect. Sutures were removed approximately 3 months after surgery.

\section{Clinical evaluation}

Clinical measurements included visual acuity, graft survival and postoperative complications. The size of the corneal lesion was reported as the equivalent circular diameter (ECD) and was calculated as previously described [15]. Corneal graft failure was defined as the irreversible loss of central graft clarity. A diagnosis of recurrent infection was made according to the constellation of characteristic clinical manifestations, such as significant epitheliopathy, new corneal infiltration, positive confocal microscopy, or positive microbial culture. Recurrent infection after lamellar keratoplasty usually started from the graft-host junction or the stromal bed. Graft rejection was diagnosed on the basis of decreased vision and stromal edema with or without anterior segment inflammation. If the cause of graft failure was considered multifactorial, the precipitating event that eventually resulted in graft failure was chosen.

\section{Statistical analysis}

Potential risk factors, including indications for grafting, the lesion size, the graft size and preoperative hypopyon, were assessed for associations with the incidence of graft failure. The statistical analyses were performed using SPSS 23.0 (SPSS Inc., Chicago, Illinois, USA) or R 3.6.0 (https://www.R-project.org/). Comparisons of continuous data among groups were performed by applying Student's $t$-test. Graft survival was evaluated by the Kaplan-Meier method, and comparisons were performed using the log-rank test. Due to the presence of nonproportional hazards between the nonviral and herpetic keratitis groups, the weighted log-rank test was used to compare the survival curves. A weighted log-rank test was carried out using the $\operatorname{comp}()$ function in the $\mathrm{R}$ package "survMisc", and Fleming-Harrington weights of $\mathrm{P}=0$, and $\mathrm{q}=0.5$ were chosen to emphasize differences later in time.

\section{Results}

\section{Patient characteristics}

Thirty-nine eligible patients (39 eyes) were implanted with APCS to treat infectious keratitis. As shown in H\&E histological stains, these replacements consisted of highly organized collagen fibers, yet no remaining cell (nuclei) were detected. SME revealed that the APCS reserved the structure of a native human corneal stroma but was less porous than the human cornea (Fig. 1).

Demographic features of the recipient are presented in Table 1 . There were 14 females and 25 males with a mean age of $48.5 \pm 13.9$ years (range, $14-72$ years). Fungal keratitis was the most common indication for surgery $(n=22$, $56.4 \%)$, and was followed by HSK $(\mathrm{n}=7,17.9 \%)$, bacterial keratitis $(\mathrm{n}=5,12.8 \%)$ and Acanthamoeba keratitis $(\mathrm{n}=4,10.3 \%)$. One case was diagnosed as fungal keratitis with concomitant bacterial infection. The size of the corneal lesions ranged from 2.78 to $7.34 \mathrm{~mm}$ (mean, $5.15 \pm 1.23 \mathrm{~mm}$ ). Descemetocele formation was observed in two patients with HSK (5.1\%). Sterile hypopyon due to intraocular inflammation rather than active intraocular infections was noted in 4 cases (10.3\%). Two of these cases were diagnosed with fungal keratitis, one case with Acanthamoeba keratitis and once case with fungal keratitis with concomitant bacterial infection. The trephination sizes of the recipients ranged from 7.25 to $8.50 \mathrm{~mm}$ (mean, $7.70 \pm 0.32 \mathrm{~mm}$ ).

\section{Visual acuity}

Corneal transparency was restored following APCS transplantation in cases without graft failure (Fig. 2). Before keratoplasty, the mean best corrected visual acuity (BCVA) was $1.38 \pm 0.94 \operatorname{logMAR}$, and only $10.3 \%(n=4)$ of the eyes had a visual acuity of $20 / 40$ or better. At the 12-month postoperative examinations, $51.2 \%(n=20)$ of the treated eyes had a visual acuity of $20 / 40$ or better, and in $85.2 \%(23 / 27)$ of the eyes that did not present graft failure, visual acuity increased by two or more Snellen lines 


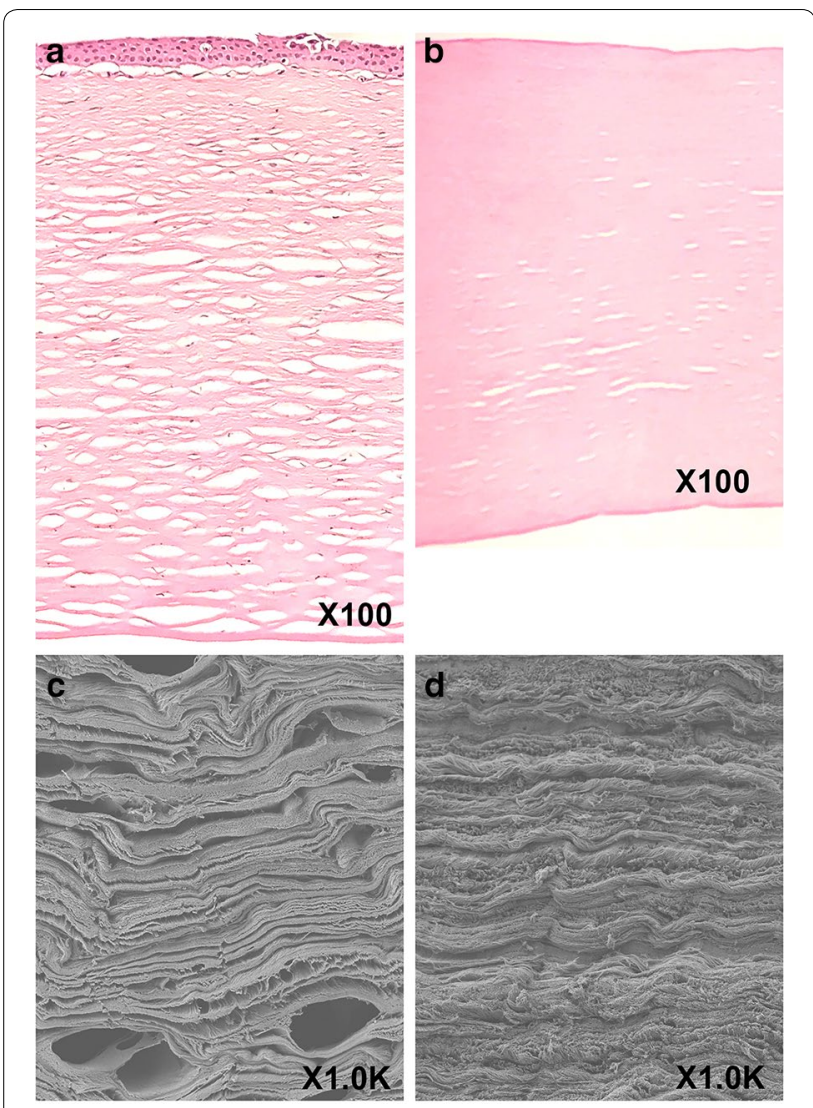

Fig. 1 Architecture of acellular porcine corneal stroma (APCS). Hematoxylin and eosin (H\&E) staining of APCS (a) and human cornea (b). Total magnification $=100 \times$. Scanning electron micrography (SEM) images of APCS (c) and human cornea (d). Total magnification $=1000 \times$

Table 1 Demographic and clinical characteristics

\begin{tabular}{ll}
\hline & Mean \pm SD (range) or $\mathbf{n}(\%)$ \\
\hline Age (years) & $45.5 \pm 13.9(14-72)$ \\
Lesion size $^{\mathrm{a}}(\mathrm{mm})$ & $5.15 \pm 1.23(2.78-7.34)$ \\
Pathogens $(\mathrm{n}=39)^{\text {Fungal keratitis }}$ & $22(56.4 \%)$ \\
HSK & $7(17.9 \%)$ \\
Bacterial keratitis & $5(12.8 \%)$ \\
Acanthamoeba keratitis & $4(10.3 \%)$ \\
Combined fungal/bacterial keratitis & $1(2.6 \%)$ \\
Descemetocele & $2(5.1 \%)$ \\
Sterile hypopyon & $4(10.3 \%)$ \\
\hline
\end{tabular}

$S D$ standard deviation, $n$ No. of eyes, $H S K$ herpes simplex keratitis, $E C D$ equivalent circular diameter

a Lesion size is expressed as the equivalent circular diameter

(Fig. 3). A few of the patients were followed up for up to 3 years. They showed good preservation of visual acuity and transparent APCS grafts (Additional file 1: Figure S1).

\section{Graft survival and risk factors}

Twelve patients presented graft failure within 1 year after keratoplasty (Fig. 4 and Table 2). Of the eyes with graft failure, two underwent repeat PKP and the remaining eyes were treated medically. Noninfectious graft melting was the major cause of graft failure (5/12, 41.7\%); the pathogeneses included extensive inflammation $(n=3)$, neovessel ingrowth $(n=1)$ and persistent epithelial defects $(n=1)$. The other causes of graft failure were recurrence of primary infection $(4 / 12,33.3 \%)$ and extensive graft neovascularization (3/12, 25\%). Recurrent fungal infection occurred within 1 month after surgery, and two of these cases occurred within the first postoperative week. The time to recurrence in HSK cases was approximately 8 months. No graft rejection occurred during the follow-up period.

The preoperative factors that influenced graft outcome are shown in Fig. 5 and Table 3. The risk was markedly higher in eyes with HSK than in eyes with nonviral keratitis [relative risk $(R R)=8.0, P=0.046$ ]. The use of grafts with a diameter equal or larger than $8.0 \mathrm{~mm}$ was also associated with a significantly higher risk of graft failure $(R R=6.5, P<0.001)$. Eyes that developed graft failure had a slightly larger corneal lesion size than eyes that achieved therapeutic success $(5.92 \pm 1.00 \mathrm{~mm}$ versus $4.81 \pm 1.17 \mathrm{~mm}, \mathrm{P}=0.007)$. Preoperative hypopyon did not present a high risk of graft failure $(\mathrm{P}=0.198)$.

\section{Discussion}

Corneal allotransplantation is a well-established technique for treating corneal blindness. However, there is a worldwide shortage of human donor corneas, and this has led to a demand for the development of biocompatible materials as an alternative [16]. Enormous progress has been made in this field, and in recent years APCS has moved into the clinical evaluation stage [10, 17-19]. A main advantage of APCS over human donor corneas is its unlimited availability. However, APCS has only been reported for therapeutic indications of keratoplasty in fungal and herpetic keratitis [10,11]. The current study further supports the viability of APCS as an alternative to the human cornea in cases of progressive infectious keratitis caused by a wide variety of pathogens, such as viruses, fungi, bacteria, and Acanthamoeba. This study is also the first to disclose the major risk factors for APCS graft failure, and this information may help in future patient selection and the postoperative management of APCS transplantation.

Corneal transplantation performed for active infectious keratitis is associated with a high risk of failure [20]. The graft survival rate varied depending on the treatment conditions [5]. For instance, in a study by Aushu et al. the therapeutic success rate of lamellar keratoplasty 


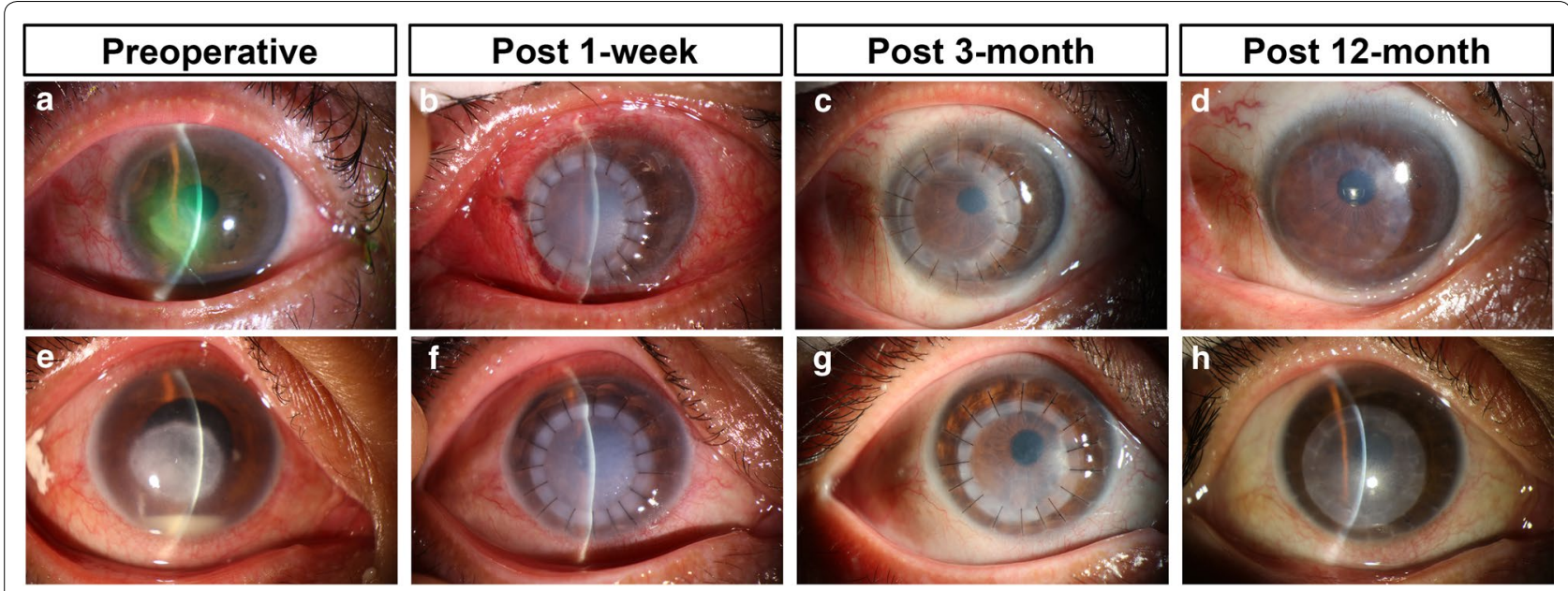

Fig. 2 Pre- and postoperative outcomes of 3 patients who underwent therapeutic lamellar keratoplasty using acellular porcine corneal stroma (APCS) for medically unresponsive infectious keratitis. Images were obtained in eyes before surgery $(\mathbf{a}, \mathbf{e})$, and at 1 day $(\mathbf{b}, \mathbf{f}), 3$ months (c, $\mathbf{g})$ and 12 months $(\mathbf{d}, \mathbf{h})$ postoperatively

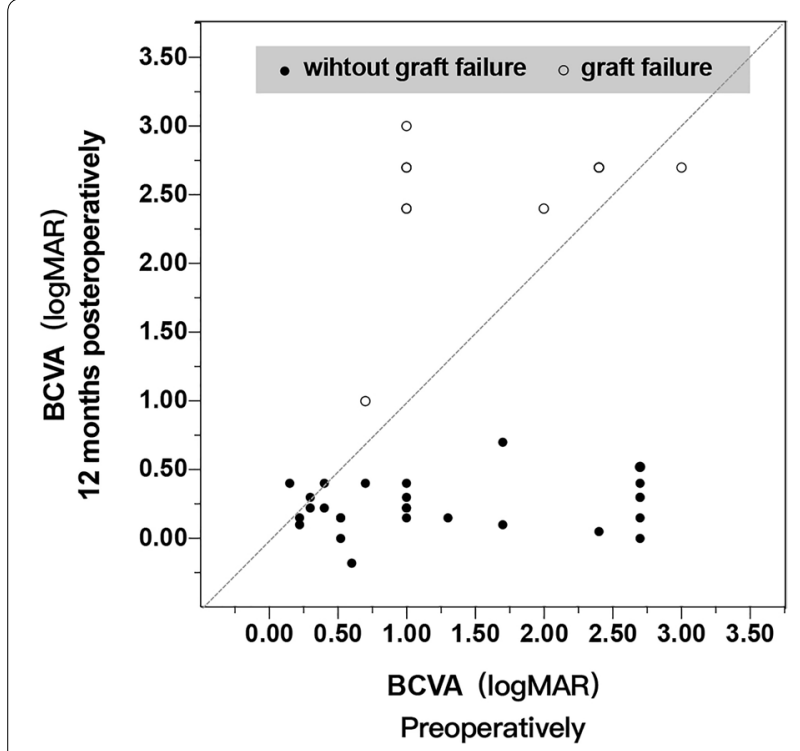

Fig. 3 Visual outcomes in eyes undergoing acellular porcine corneal stroma (APCS) transplantation. The majority of patients had improved best-corrected visual acuity (BCVA) at 12 months after surgery.

The points on the diagonal line show that the BCVA was the same preoperatively and 12 months postoperatively. Counting fingers, hand motion and light perception are shown as logarithm of the minimum angle of resolution (logMAR) values of $0.004,0.002$ and 0.001 , respectively

performed using a human cornea was $86.6 \%$ in nonviral infectious keratitis [4], and Zhang et al. reported that APCS transplantation achieved therapeutic success in $87.2 \%(41 / 47)$ of fungal keratitis cases [10], indicating that the rate of success was comparable between human donor corneas and APCS. In our study, the therapeutic success rate of APCS transplantation in nonviral infectious keratitis was $75 \%(24 / 32)$, and this rate was a slightly lower than that reported by Zhang et al. The severity of corneal infection might contribute to the differences observed between these two studies. In addition, visual rehabilitation is a secondary consideration in therapeutic keratoplasty. Both previous studies and this study showed that APCS transplantation can restore the optical clarity of the cornea, and the majority of recipients without graft failure eventually had improved visual acuity [10]. Therefore, all of these results collectively suggest that APCS meets the clinical demands for xenografts in various types of infectious keratitis.

Graft rejection is always the primary concern in xenotransplantation. While APCS implantation is by its nature a xenotransplantation, no evidence of graft rejection was observed in any of our patients at the last follow-up. Our findings are consistent with those presented in previous reports $[10,21]$. Fresh porcine corneal stroma contributes only $1.62 \%$ and $6.12 \%$, respectively, of the cellular and humoral immunogenicity of an intact cornea [22]. During the process of tissue decellularization, cellular material and antigen molecules are nearly completely removed, significantly alleviating the host immune response following its transplantation [21]. A previous study showed that transplantation of APCS into corneal pockets and subcutaneous tissue was incapable of triggering an accepted immune response [13]. Indeed, a significant advantage of APCS is its inherently low antigenicity and immunogenicity. However, it is worth noting that collagen retains weak antigenic determinants in its telopeptide regions. Moreover, APCS contains, in addition to collagen, a very few small number of antigenic 

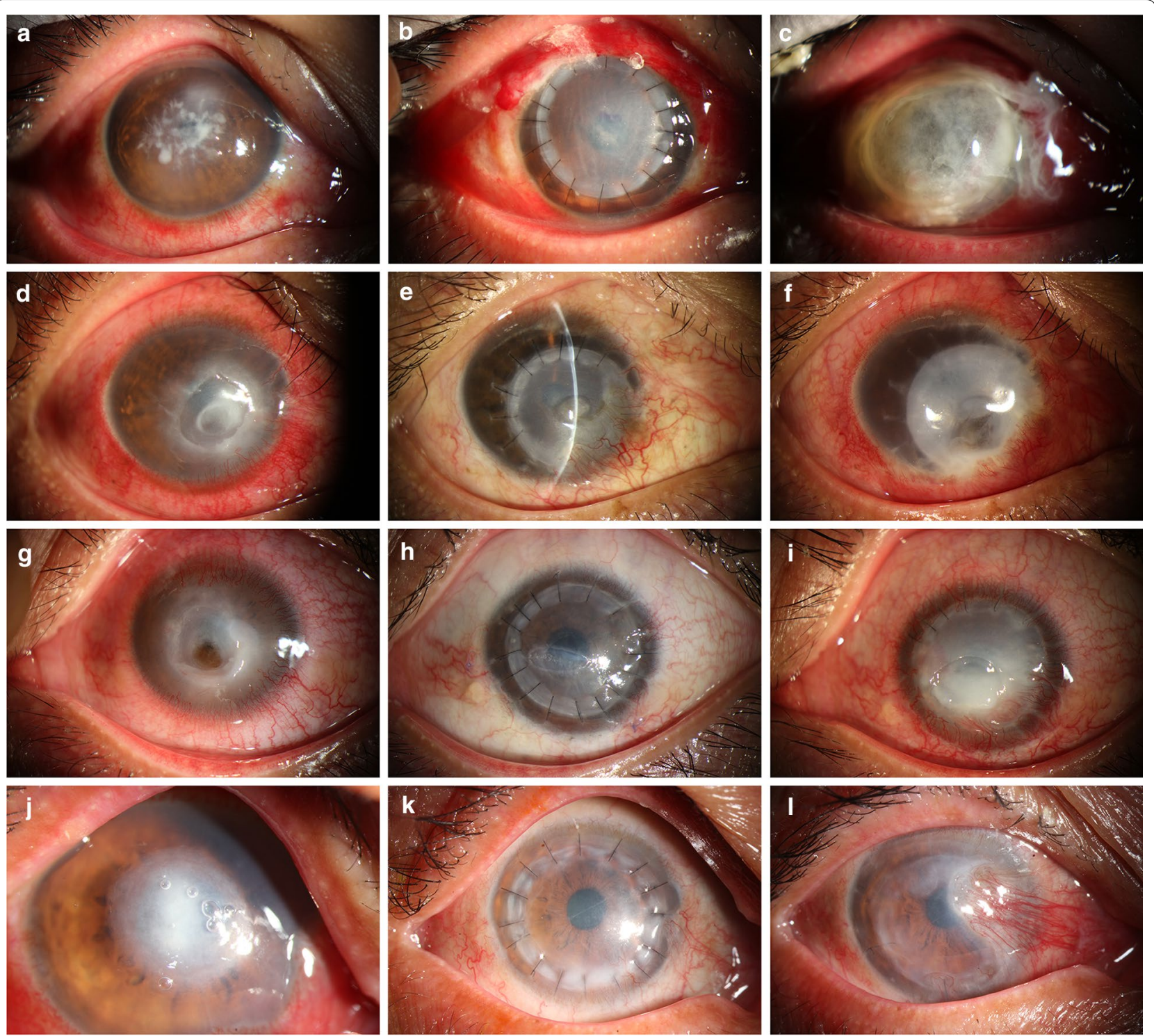

Fig. 4 Slit-lamp microscopy images of eyes with graft failure. a-c A patient was diagnosed with fungal keratitis who experienced recurrent infection within 1 week after the operation. A repeated PKP was performed. $\mathbf{d}-\mathbf{f}$ A patient with mild eyelid deformity and lagophthalmos, which resulted in neovessel ingrowth and led to late graft dissolution. g-i A patient with a diagnosis of herpes simplex keratitis who had persistent epithelial defects after the operation that ultimately progressed to corneal ulceration. $\mathbf{j}$-I The graft was transparent in the initial postoperative period, but neovessels later grew into the center of the graft

noncollagenous proteins and residual cell-associated components [23]. In this regard, although no host-versus-graft type rejection was observed in this or previous studies, anti-rejection therapy should be routinely postoperatively administered to APCS-implanted eyes. Nevertheless, the duration of anti-rejection medication requires further investigation.

In our study, five patients developed noninfectious graft melting, which is the most common cause of graft failure. Graft melting is a devastating complication of corneal transplantation. It is a destructive process involving collagen dissolution driven by the excessive release of tissue collagenase [24]. One group of these collagenases is the matrix metalloproteinases (MMPs) [25]. Graft melting occurs under a number of conditions, including infectious or sterile inflammation. Under sterile conditions, such as intense inflammation, persistent corneal epithelial defect and corneal neovascularization, proinflammatory cytokines are released, resulting in increased collagenases activity 
Table 2 Clinical characteristics of patients with graft failure

\begin{tabular}{|c|c|c|c|c|c|}
\hline Cause of graft failure & Indications & Lesion size ${ }^{\mathbf{b}}(\mathrm{mm})$ & Graft size $(\mathrm{mm})$ & $\begin{array}{l}\text { Graft survival time } \\
\text { (months) }\end{array}$ & Prognosis \\
\hline \multicolumn{6}{|l|}{ Infection recurrence } \\
\hline 1 & Fungal & 3.39 & 7.50 & 0.2 & Repeated PK \\
\hline 2 & Fungal & 6.01 & 8.50 & 0.1 & Repeated PK \\
\hline 3 & Fungal & 6.51 & 7.75 & 0.5 & Controlled \\
\hline 4 & HSK & 5.80 & 8.00 & 8.0 & Controlled \\
\hline \multicolumn{6}{|l|}{ Graft melting ${ }^{a}$} \\
\hline $5^{c}$ & HSK & 7.18 & 8.00 & 2.2 & Controlled \\
\hline $6^{d}$ & HSK & 5.36 & 7.75 & 3.0 & Controlled \\
\hline $7^{d}$ & HSK & 7.16 & 8.25 & 1.0 & Controlled \\
\hline $8^{d}$ & Acanthamoeba & 5.60 & 8.25 & 4.3 & Repeated PK \\
\hline $9^{e}$ & Fungal & 5.89 & 7.75 & 5.0 & Repeated PK \\
\hline \multicolumn{6}{|c|}{ Extensive graft neovascularization } \\
\hline 10 & Fungal & 6.22 & 8.00 & 6.5 & Controlled \\
\hline 11 & Fungal & 5.42 & 8.50 & 6.5 & Controlled \\
\hline 12 & Bacterial & 6.52 & 7.25 & 6.0 & Controlled \\
\hline
\end{tabular}

HSK herpetic simplex viral keratitis, $P K$ penetration keratoplasty

a Noninfectious graft melting

b Lesion size reported as the equivalent circular diameter

c Graft melting caused by persistent epithelial defect

d Graft melting caused by extensive inflammation

e Graft melting caused by neovessel ingrowth
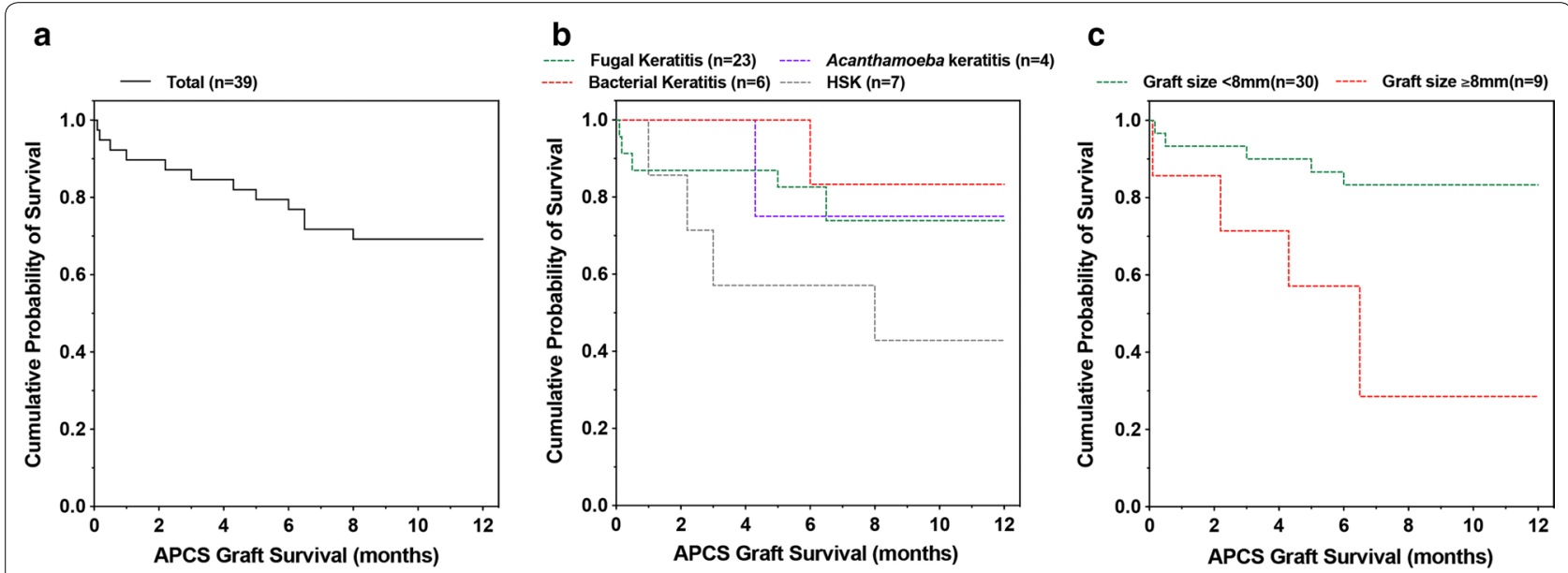

Fig. 5 Kaplan-Meier survival curve depicting the graft failure episodes in eyes submitted to acellular porcine corneal stroma (APCS) implantation. a Total graft survival; $\mathbf{b}$ graft survival in eyes with various types of infectious keratitis; $\mathbf{c}$ graft survival in eyes with a graft size larger or smaller than a diameter of $8 \mathrm{~mm}$. HSK, herpes simplex keratitis

and subsequent graft melting [26-28]. Collagen-based corneal stroma substitutes are generally more prone than human donor corneas to collagenase dissolution [29]. However, APCS preserves the interwoven lamellar structure of the natural cornea, and this endows it with better resistance to collagenase than is achieved by the biosynthetic collagen materials. Therefore, graft melting does not seem to be an overly concerning issue with regard for use of APCS.

Several preoperative factors that exerted a profound impact on graft survival were identified in this study. First, we noted that APCS transplantation had a much worse prognosis in clinically active herpetic eyes than in eyes with nonviral microbial keratitis. In our series, the 
Table 3 Possible risk factors for graft failure

\begin{tabular}{|c|c|c|c|c|}
\hline Parameter & Therapeutic success $(n=27)$ & Graft failure $(n=12)$ & $\mathbf{P}$ & Type of statistical test \\
\hline Indication for graft & & & 0.046 & Weighted log-rank test ${ }^{b}$ \\
\hline Nonviral & $24(75.0 \%)$ & $8(25.0 \%)$ & & \\
\hline Herpetic & $3(42.9 \%)$ & $4(57.1 \%)$ & & \\
\hline Mean lesion size ${ }^{a}(\mathrm{~mm})$ & $4.81 \pm 1.17(2.78-7.34)$ & $5.92 \pm 1.00(3.39-7.18)$ & 0.007 & $t$-test \\
\hline Mean graft size (mm) & $7.59 \pm 0.21(7.25-8.00)$ & $7.95 \pm 0.38(7.25-8.5)$ & $<0.001$ & t-test \\
\hline Graft size group & & & $<0.001$ & Log-rank test \\
\hline$<8.0$ & $25(83.3 \%)$ & $5(16.7 \%)$ & & \\
\hline$\geq 8.0$ & $2(22.2 \%)$ & $7(77.8 \%)$ & & \\
\hline Preoperative sterile hypopyon & & & 0.198 & Log-rank test \\
\hline Absence & $23(65.7 \%)$ & $12(34.3 \%)$ & & \\
\hline Presence & $4(100 \%)$ & $0(0 \%)$ & & \\
\hline
\end{tabular}

a Lesion size reported as the equivalent circular diameter

b Because of the presence of nonproportional hazards, the weighted log-rank test was used to compare the survival distributions between nonviral keratitis and herpetic keratitis groups. Fleming-Harrington weights of $P=0, q=0.5$ were set to emphasize differences occurring later in time

1-year graft survival rate for cases of bacterial, fungal and Acanthamoeba keratitis varied from 73.9 to $85.7 \%$; in contrast, only $42.9 \%(3 / 7)$ of our patients with HSK achieved therapeutic success. The extremely low survival rate of APCS grafts in active herpetic keratitis observed in this study is consistent with previous reports. For example, Zhang et al. performed APCS transplantation to treat 13 cases of herpes simplex virus (HSV)-related corneal ulcers or scarring [11] and observed graft failure in $15 \%$ of quiescent eyes but up to $71.43 \%$ (5/7) of inflamed eyes during the follow-up period. Therefore, similar to human donor cornea, APCS transplantation is not recommended in patients with clinically active HSK.

However, under some conditions, such as medically uncontrolled ulceration, ulceration at risk of perforation, and insufficient fresh donor corneas to meet demands, APCS transplantation may be applied. There are some management considerations that could improve surgical prognosis. HSK is typically characterized by recurrent episodes of infections, because HSV can be latent in the trigeminal ganglion [30]. After keratoplasty, HSV is able to migrate into the graft along with the regenerated subbasal nerve fibers [11, 31]. HSK patients may therefore remain at risk of recurrent infection beyond the initial postoperative period. Sufficient antiviral courses are therefore necessary in these patients. Moreover, in the present study, we found that noninfectious graft melting was the most common cause of graft failure in HSK patients. Possible recognized predisposing factors for APCS graft melting in HSK patients include ongoing ocular surface inflammation and a persistent epithelial defect. A previous study revealed that early postoperative persistent epithelial defects were present in $8 \%$ of HSK cases that underwent PK and $44 \%$ of cases that underwent LK [32]. These inflammatory settings could result in elevated levels of collagenases such as MMP, being produced by inflammatory cells, predominantly including neutrophils [26-28]. Therefore, in this regard, the prevention of corneal epithelial defects and efficient anti-inflammatory medication are particularly necessary to achieve therapeutic success in eyes undergoing APCS transplantation for active HSK.

In our study, another preoperative factor associated with the incidence of graft failure was graft size. We found that APCS grafts performed with a size $\geq 8.0 \mathrm{~mm}$ had a higher risk than smaller grafts of developing recurrent infection ( $22.2 \%$ vs $6.7 \%)$, graft melting (33.3\% vs $6.7 \%)$ or extensive graft neovascularization $(22.2 \%$ vs 3.3\%). Williams et al. and Volker-Dieben et al. conducted a large retrospective analysis to evaluate the effect of graft size on graft outcomes. Both of these authors showed that graft failure rates were higher for grafts exceeding of $8.0 \mathrm{~mm}[33,34]$. In therapeutic PKP for infectious keratitis, Killingsworth also reported that graft size significantly influenced therapeutic success and that survival was better for smaller grafts $(<9.0 \mathrm{~mm})$ than larger grafts $(\geq 9.0 \mathrm{~mm})$ in bacterial $(83 \%$ vs $50 \%)$ and fungal $(75 \%$ vs $33 \%)$ keratitis [35]. The original size of the APCS grafts used in this study was $10 \mathrm{~mm}$, the APCS grafts were trephined into the size of choice during keratoplasty. In our study, trephines of 7.25 to $8.25 \mathrm{~mm}$ in diameter were used, and these sizes were larger than that those used in the recently published study by Zhang and colleagues $(6.25-7.50 \mathrm{~mm})$ [11]. We found that APCS grafts performed with a size $\geq 8.0 \mathrm{~mm}$ resulted in a much worse survival rate than was achieved in for smaller grafts (83.33\% vs $11.11 \%, R R=6.5)$. Two explanations might be considered for this findings: (a) the use of a large 
graft might increase the chance of an immune response derived from the corneal limbus; and (b) a larger graft size might also indicate the presence of more several preoperative infections [36].

Moreover, we found that compared to human corneal transplantation, in APCS transplantation, the graft size seemed to exert a more significant influence on graft failure. We suggest that this difference could be due to two reasons. First, as we discussed above, the use of a large graft might increase the chance that the graft will interact with immune cells derived from the corneal limbus. Even worse, APCS grafts are more prone than human donor corneas to interact with collagenase released by immune cells than donor human cornea [29]. Second, because it is a xenotransplantation product, the residual antigenicity of APCS should not be overlooked [23]. If a graft with a larger size is used, APCS grafts might have a higher risk of presenting antigens to limbal immune cells and thereby inducing an immune response. Therefore, according to our findings, the use of APCS with a diameter larger than $8.0 \mathrm{~mm}$ should potentially be avoided.

A relatively short follow up period is a limitation of this study. Because serious complications, such as recurrent infectious and graft rejection, are more likely to occur within 1 year after corneal transplantation, our patients were followed up for at least 12 months. A future study will be designed to verify the long-term success of APCS transplantation for infectious keratitis.

\section{Conclusion}

Based on these findings, although the number of patients was limited and the follow-up duration was short, this study provides some insights that will be valuable for the future application of APCS, including surgical indications and postoperative management.

\section{Supplementary information}

Supplementary information accompanies this paper at https://doi. org/10.1186/s12967-019-02192-z.

Additional file 1: Figure S1. Slit-lamp biomicroscopy performed at 2 (a) and 3 (b) years after implantation with acellular porcine corneal stroma (APCS). The implant was well integrated into the recipient cornea. The patient had a visual acuity of 20/25 in the operated eye.

\section{Abbreviations}

PKP: penetrating keratoplasty; ALK: anterior lamellar keratoplasty; APCS: acellular porcine corneal stroma; DM: Descemet's membrane; HSK: herpes simplex keratitis; PBS: phosphate-buffered saline; H\&E: hematoxylin and eosin; SEM: scanning electron microscopy; DALK: deep anterior lamellar keratoplasty; ECD: equivalent circular diameter; BCVA: best corrected visual acuity; MMP: matrix metalloproteinase; HSV: herpes simplex virus.

Acknowledgements

Not applicable.

\section{Authors' contributions}

All authors read and approved the final manuscript. JY was the principal study investigator for this study and participated in the study design, surgery performance and manuscript revision. SL and ML participated in data analysis and drafting the manuscript. LG participated in the execution of statistical analyses. LP and BW participated in the histological examination of APCS. YD, $J Z$, QW and YX participated in following up the patients. All authors read and approved the final manuscript.

\section{Funding}

This work was supported by the National Natural Science Foundation of China [Grant Number 81870633]. The funder was not involved in the execution of the research or the preparation of the manuscript.

\section{Availability of data and materials}

All data generated or analyzed during this study are included in this published article.

\section{Ethics approval and consent to participate}

This study adhered to the tenets of the Declaration of Helsinki and was approved by the Ethics Committee of Zhongshan Ophthalmic Center of Sun Yat-sen University. All patients provided written consent to participate in this study.

\section{Consent for publication}

Not applicable.

\section{Competing interests}

The authors declare that they have no competing interests.

Received: 16 Auqust 2019 Accepted: 23 December 2019

Published online: 30 December 2019

\section{References}

1. Hussain S, Johri A, Rao SK. Infectious keratitis: a review. Bengal Ophthal J. 2015;1-11. http://oswb.org/wp-content/uploads/2017/08/OSWB_Journ al_Nov_2015.pdf.

2. Whitcher JP, Srinivasan M, Upadhyay MP. Corneal blindness: a global perspective. Bull World Health Org. 2001;79:214-21.

3. Austin A, Lietman T, Rose-Nussbaumer J. Update on the management of infectious keratitis. Ophthalmology. 2017;124:1678-89.

4. Anshu A, Parthasarathy A, Mehta JS, Htoon HM, Tan DT. Outcomes of therapeutic deep lamellar keratoplasty and penetrating keratoplasty for advanced infectious keratitis: a comparative study. Ophthalmology. 2009;116:615-23.

5. Sharma N, Sachdev R, Jhanji V, Titiyal JS, Vajpayee RB. Therapeutic keratoplasty for microbial keratitis. Curr Opin Ophthalmol. 2010;21:293-300.

6. Shang $X$, Zhang M. Body and organ donation in Wuhan, China. Lancet. 2010;376:1033-4

7. Hicks C, Crawford G, Lou X, Tan D, Snibson G, Sutton G, Downie N, Werner $\mathrm{L}$, Chirila T, Constable I. Corneal replacement using a synthetic hydrogel cornea, AlphaCor: device, preliminary outcomes and complications. Eye. 2003;17:385-92.

8. Liu Y, Gan L, Carlsson DJ, Fagerholm P, Lagali N, Watsky MA, Munger $R$, Hodge WG, Priest D, Griffith M. A simple, cross-linked collagen tissue substitute for corneal implantation. Invest Ophthalmol Vis Sci. 2006:47:1869-75.

9. Matthyssen S, Van den Bogerd B, Dhubhghaill SN, Koppen C, Zakaria N. Corneal regeneration: a review of stromal replacements. Acta Biomater. 2018;69:31-41.

10. Zhang MC, Liu X, Jin Y, Jiang DL, Wei XS, Xie HT. Lamellar keratoplasty treatment of fungal corneal ulcers with acellular porcine corneal stroma. Am J Transplant. 2015;15:1068-75.

11. Zheng J, Huang X, Zhang Y, Wang Y, Qin Q, Lin L, Jin X, Lam C, Zhang J. Short-term results of acellular porcine corneal stroma keratoplasty for herpes simplex keratitis. Xenotransplantation. 2019;26:e12509. 
12. Lynch AP, Ahearne M. Strategies for developing decellularized corneal scaffolds. Exp Eye Res. 2013;108:42-7.

13. Luo H, Lu Y, Wu T, Zhang M, Zhang Y, Jin Y. Construction of tissueengineered cornea composed of amniotic epithelial cells and acellular porcine cornea for treating corneal alkali burn. Biomaterials. 2013;34:6748-59.

14. Xu CC, Chan RW. Pore architecture of a bovine acellular vocal fold scaffold. Tissue Eng Part A. 2008;14:1893-903.

15. Premaladha J, Ravichandran K. Novel approaches for diagnosing melanoma skin lesions through supervised and deep learning algorithms. J Med Syst. 2016:40:96.

16. Whitcher JP, Srinivasan M, Upadhyay M. Corneal blindness: a global perspective. J Bull World Health Org. 2001;79:214-21.

17. Liu W, Merrett K, Griffith M, Fagerholm P, Dravida S, Heyne B, Scaiano JC, Watsky MA, Shinozaki N, Lagali N, et al. Recombinant human collagen for tissue engineered corneal substitutes. Biomaterials. 2008;29:1147-58.

18. Gil ES, Mandal BB, Park SH, Marchant JK, Omenetto FG, Kaplan DL. Helicoidal multi-lamellar features of RGD-functionalized silk biomaterials for corneal tissue engineering. Biomaterials. 2010;31:8953-63.

19. Yoeruek E, Bayyoud T, Maurus C, Hofmann J, Spitzer MS, Bartz-Schmidt KU, Szurman P. Decellularization of porcine corneas and repopulation with human corneal cells for tissue-engineered xenografts. Acta Ophthalmol. 2012;90:e125-31.

20. Di Zazzo A, Kheirkhah A, Abud TB, Goyal S, Dana R. Management of highrisk corneal transplantation. Surv Ophthalmol. 2017;62:816-27.

21. Hara H, Cooper DKJX. The immunology of corneal xenotransplantation: a review of the literature. Xenotransplantation. 2010;17:338-49.

22. Wang Z, Ge J, Xu J, Chen J. Relative quantitative analysis of corneal immunogenicity. Zhonghua Yan Ke Za Zhi. 2002;38:535-8.

23. Lynn AK, Yannas IV, Bonfield W. Antigenicity and immunogenicity of collagen. J Biomed Mater Res B Appl Biomater. 2004;71:343-54.

24. Jin B, Zhu X. The pathogenesis and prevention of corneal graft melting after keratoplasty. J Clin Ophthalmol. 2017:1:10-8.

25. Hossain P. The corneal melting point. Eye (Lond). 2012;26:1029-30.

26. Carter RT, Kambampati R, Murphy CJ, Bentley E. Expression of matrix metalloproteinase 2 and 9 in experimentally wounded canine corneas and spontaneous chronic corneal epithelial defects. Cornea. 2007;26:1213-9.
27. Shi W, Liu J, Li M, Gao H, Wang T. Expression of MMP, HPSE, and FAP in stroma promoted corneal neovascularization induced by different etiological factors. Curr Eye Res. 2010;35:967-77.

28. Kvanta A, Sarman S, Fagerholm P, Seregard S, Steen B. Expression of matrix metalloproteinase-2 (MMP-2) and vascular endothelial growth factor (VEGF) in inflammation-associated corneal neovascularization. Exp Eye Res. 2000:70:419-28.

29. Angele P, Abke J, Kujat R, Faltermeier H, Schumann D, Nerlich M, Kinner B, Englert C, Ruszczak Z, Mehrl R, Mueller R. Influence of different collagen species on physico-chemical properties of crosslinked collagen matrices. Biomaterials. 2004;25:2831-41.

30. Bastian F, Rabson A, Yee C, Tralka T. Herpesvirus hominis: isolation from human trigeminal ganglion. Science. 1972;178:306-7.

31. Lyall DA, Tarafdar S, Gilhooly MJ, Roberts F, Ramaesh K. Long term visual outcomes, graft survival and complications of deep anterior lamellar keratoplasty in patients with herpes simplex related corneal scarring. Br J Ophthalmol. 2012;96:1200-3.

32. Gutow A, Soong HK, Meyer RF, Sugar A, Musch DC. Corneal epithelial healing after keratoplasty for herpes simplex keratitis. Cornea. 1986;5:5-10.

33. Williams KA, Roder D, Esterman A, Muehlberg SM, Coster DJ. Factors predictive of corneal graft survival. Report from the Australian corneal graft registry. Ophthalmology. 1992;99:403-14.

34. Volker-Dieben HJ, D'Amaro J, Kok-van Alphen CC. Hierarchy of prognostic factors for corneal allograft survival. Aust N Z J Ophthalmol. 1987;15:11-8.

35. Killingsworth DW, Stern GA, Driebe WT, Knapp A, Dragon DM. Results of therapeutic penetrating keratoplasty. J Ophthalmol. 1993;100:534-41.

36. Kashiwabuchi RT, de Freitas D, Alvarenga LS, Vieira L, Contarini P, Sato E, Foronda A, Hofling-Lima AL. Corneal graft survival after therapeutic keratoplasty for Acanthamoeba keratitis. Acta Ophthalmol. 2008;86:666-9.

\section{Publisher's Note}

Springer Nature remains neutral with regard to jurisdictional claims in published maps and institutional affiliations.
Ready to submit your research? Choose BMC and benefit from:

- fast, convenient online submission

- thorough peer review by experienced researchers in your field

- rapid publication on acceptance

- support for research data, including large and complex data types

- gold Open Access which fosters wider collaboration and increased citations

- maximum visibility for your research: over $100 \mathrm{M}$ website views per year

At BMC, research is always in progress.

Learn more biomedcentral.com/submissions 